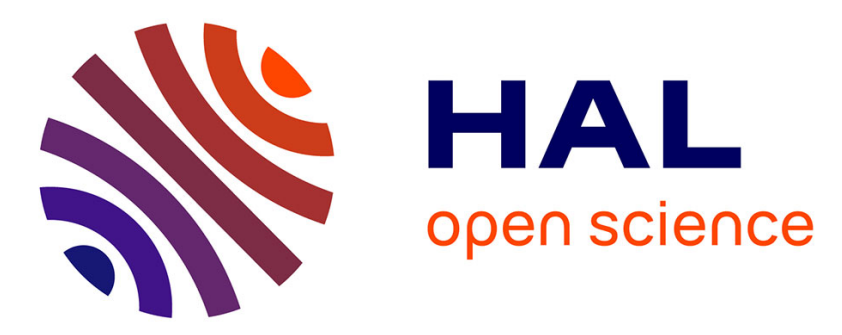

\title{
Relativistic quantum chemical calculations show that the uranium molecule U2 has a quadruple bond
}

\author{
Stefan Knecht, Hans Jørgen Aagaard Jensen, Trond Saue
}

\section{To cite this version:}

Stefan Knecht, Hans Jørgen Aagaard Jensen, Trond Saue. Relativistic quantum chemical calculations show that the uranium molecule U2 has a quadruple bond. Nature Chemistry, 2019, 11 (1), pp.40-44. 10.1038/s41557-018-0158-9 . hal-01973244

\section{HAL Id: hal-01973244 \\ https://hal.science/hal-01973244}

Submitted on 28 Jan 2020

HAL is a multi-disciplinary open access archive for the deposit and dissemination of scientific research documents, whether they are published or not. The documents may come from teaching and research institutions in France or abroad, or from public or private research centers.
L'archive ouverte pluridisciplinaire HAL, est destinée au dépôt et à la diffusion de documents scientifiques de niveau recherche, publiés ou non, émanant des établissements d'enseignement et de recherche français ou étrangers, des laboratoires publics ou privés. 


\section{Relativistic Quantum Chemical Calculations Show that the Uranium Molecule $\mathbf{U}_{2}$}

\section{Has a Quadruple Bond}

Stefan Knecht*, 1, a) Hans Jørgen Aa. Jensen, ${ }^{2}$ and Trond Saue ${ }^{3,4}$

1) Laboratorium für Physikalische Chemie, ETH Zürich, Vladimir-Prelog-Weg 2, CH-8093 Zürich, Schweiz

2) Department of Physics, Chemistry and Pharmacy, University of Southern Denmark, DK-5230 Odense M, Denmark

${ }^{3)}$ Laboratoire de Chimie et Physique Quantiques, IRSAMC,

Université Paul Sabatier Toulouse III, 118 Route de Narbonne, F-31062 Toulouse, France

4) Centre for Advanced Study at the Norwegian Academy of Science and Letters, Drammensveien 78, N-0271 Oslo, Norway

\footnotetext{
a)Electronic mail: stefan.knecht@phys.chem.ethz.ch
} 


\begin{abstract}
Our understanding of the bonding, reactivity and electronic structure of actinides is lagging behind the rest of the periodic table. This can be partly explained by the challenges that one faces in studying such radioactive compounds experimentally and also by the need to properly account for relativistic effects in theoretical studies. A further challenge is the very complicated electronic structures encountered in actinide chemistry, as vividly illustrated by the naked diuranium molecule $\mathrm{U}_{2}$. Here we report a computational study of this emblematic molecule using state-of-the-art relativistic quantum chemical methods. Notably, the variational inclusion of spin-orbit interaction leads to not only a different electronic ground state, but also a lower bond multiplicity compared to previous studies.
\end{abstract}


Understanding the nature of a chemical bond ${ }^{1}$ and how it relates to the chemical reactivity of a molecule is of central interest in chemistry. Despite recent efforts, ${ }^{2-12}$ insight into the bonding of the $5 f$ elements is still limited, in particular with respect to a basic understanding of which atomic orbitals contribute and the degree of covalency in actinide-ligand bonding. ${ }^{13}$ Concerning the most prominent $5 f$ element uranium, ${ }^{14}$ recent works have explored the nature of supported $U-U^{15-20}$ as well as uranium-ligand (multiple) bonds ${ }^{21-25}$ and their implications on the physical and chemical properties of the corresponding complexes. ${ }^{26-29}$ In contrast, still today comparatively little is known about the chemical bonding in the fundamental, naked diuranium molecule $\mathrm{U}_{2}{ }^{30-33}$ which was experimentally first observed in the early 1970s. ${ }^{34}$ In 2005 Laura Gagliardi and Björn Roos presented a computational study which concluded that diuranium is a stable molecule with a very complicated bonding pattern, featuring all known covalent bonding types and leading to a quintuple bond. ${ }^{32}$ Although their study did include relativistic effects, Gagliardi and Roos did not fully explore the effect of spin-orbit (SO) interaction on the nature of the ground state and bonding.

What makes the early actinides and in particular uranium so unique within the periodic table of elements is the extended set of valence electronic shells that are available for bonding. The key valence atomic orbitals (AOs) of uranium are the seven $5 f$, the five $6 d$ and the $7 s$ orbitals which are all (partially) occupied in the ground state $[\mathrm{Rn}] 5 f^{3} 6 d^{1} 7 s^{2}$ electronic configuration of the $\mathrm{U}$ atom. However, as illustrated by Fig. 1, the availability of the $5 f$ orbitals and an increased availability of the $6 d$ orbitals for bonding is very much a relativistic effect. Two kinds of relativistic effects are commonly distinguished, ${ }^{35-37}$ namely scalar-relativistic effects and SO interaction. The former arises from the high speed and associated relativistic mass increase of electrons in the vicinity of heavy nuclei, whereas the latter originates from the interaction of electron spin with the magnetic field induced by charged particles (nuclei and other electrons) in relative motion and leads to a coupling of spin and spatial degrees of freedom. ${ }^{38}$ Both relativistic effects profoundly modify energetics (see Figure 1) and the spatial extent of AOs and hence molecular bonding and structure, ${ }^{37}$ with spin-orbit effects becoming particularly noticeable from sixth-row elements and onwards due to second-order effects (orbital relaxation). ${ }^{39,40}$ As summarized in Supplementary Table 1, the spatial extent of the frontier AOs - measured by 
the root-mean-square ( $\mathrm{rms}$ ) radius of the associated radial function — is, similar to the energetic considerations above, subject to distinct relativistic effects. Scalar-relativistic effects are most pronounced, leading to an increase of the rms values for the $5 f(6 d)$ orbitals by $15 \%$ (14\%), thus allowing for better spatial overlap upon bond formation in the molecule, in particular since the $7 s$ undergoes a significant contraction with inclusion of scalar-relativistic effects, as underlined by a decrease in the rms value by $14 \%$. SO effects then further increase/decrease the rms radii of the radial functions for the spin-orbit split $6 d$ and $5 f$ orbitals by approximately $\pm 2 \%$.

In their seminal work, Gagliardi and $\operatorname{Roos}^{32}$ presented the first computational evidence that $\mathrm{U}_{2}$ is a stable molecule with a binding energy of $40.2 \mathrm{kcal} / \mathrm{mol}$ at the scalar-relativistic 2nd-order complete active space perturbation theory (CASPT2) level, being reduced to $30.5 \mathrm{kcal} / \mathrm{mol}$ upon the perturbative inclusion of SO interaction. Interestingly, a promotion energy of $17.9 \mathrm{kcal} / \mathrm{mol}$ is required per atom to go from the $5 f^{3} 6 d^{1} 7 s^{2}{ }^{5} \mathrm{~L}_{6}$ ground state to the excited $5 f^{3} 6 d^{2} 7 s^{1}{ }^{7} \mathrm{M}_{6}$ state, ${ }^{41}$ the lowest excited level with unpaired $7 s$ electrons, thus making $7 s$ available for bonding and avoiding Pauli repulsion between closed $7 s$ shells. Excitations out of the $5 f$ orbitals takes considerably more energy; the first such state, a $5 f^{2} 6 d^{2} 7 s^{2}{ }^{5} \mathrm{~L}_{6}$ state, lies $32.9 \mathrm{kcal} / \mathrm{mol}$ above the atomic ground state. The calculated binding energies for $\mathrm{U}_{2}$ may be compared to the much larger energy of $52 \pm 5$ $\mathrm{kcal} / \mathrm{mol}$ obtained from experimental mass spectrometry data. ${ }^{34}$ However, the experiment was carried out at high temperature $\left(2500-2700{ }^{\circ} \mathrm{C}\right)$ and the dissociation energy was extracted assuming an equilibrium $\mathrm{U}$ - $\mathrm{U}$ internuclear distance of $\mathrm{R}_{e}=3.0 \AA$ and a vibrational frequency $\omega_{e}=100 \mathrm{~cm}^{-1}$, leading the authors themselves to suggest that their value is somewhat overestimated.

In their first paper, Gagliardi and Roos report a spin-free electronic ground state ${ }^{7} \mathrm{O}_{\mathrm{g}}$, which corresponds to a septet state $(S=3)$ with projection $\Lambda=11$ of the total electronic angular momentum on the internuclear axis, and suggest that the $\Omega=14$ component will be the ground state upon inclusion of SO coupling. However, in a subsequent publication an excited state of $\Omega=9_{\mathrm{g}}(\Lambda=12)$ was reported a mere $80 \mathrm{~cm}^{-1}$ above a $\Omega=8_{\mathrm{g}}(\Lambda=11)$ ground state, such that it was not possible to unequivocally determine the ground state of $\mathrm{U}_{2} \cdot{ }^{33}$ Note that the main text of Ref. 33 reports on page 17004 the calculated first excited state as $\Omega=8_{\mathrm{g}}(\Lambda=12)$ but we base our designation $\Omega=9_{\mathrm{g}}(\Lambda=12)$ on 
Table 6 in the same paper. The bonding picture in the electronic ground state as determined from their scalar-relativistic complete-active space self-consistent field (CASSCF) calculations $^{32,33}$ can be summarized as follows: Three "normal" two-electron two-center bonds, one of $\sigma_{\mathrm{g}}$ and two of $\pi_{\mathrm{u}}$ symmetry, are formed with predominant $7 \mathrm{~s}$ and $6 \mathrm{~d}$ atomic character, respectively, together with four one-electron two-center bonds, of symmetries $\sigma_{\mathrm{g}}, \pi_{\mathrm{u}}$, and $\delta_{\mathrm{g}}(2)$ comprising contributions from $6 d$ and $5 f$ AOs. In addition, two ferromagnetically coupled $5 f_{\phi}$-electrons are localized in nonbonding molecular orbitals (MOs) on each $\mathrm{U}$ center. Interestingly, exchange stabilization effects arising from interactions of the localized $5 f_{\phi}$-electrons with the one-electron bonds are found to exceed the energetic benefits of an antiferromagnetically coupling of the localized $5 f_{\phi}$-electrons. The proposed bonding picture in $\mathrm{U}_{2}$ corresponds undoubtedly to one of the most complex electronic structures hitherto known for any two atoms in a molecule.

Discussing chemical bonding in $\mathrm{U}_{2}$ requires the definition of an intuitive and stable measure that allows us to characterize possible multiple bonds in a distinct manner. Many such quantities to describe a chemical bond exist and have been applied to explorations of the chemical bonding in $f$-element complexes, see for example Ref. 3. One such scheme is based on the concept of bond order, defined as half the difference between the number of bonding and anti-bonding electrons in a molecule ${ }^{42}$. It was extended to multiconfigurational wave functions by Roos and co-workers ${ }^{32,33,43}$ through the introduction of an effective bond order (EBO)

$$
\mathrm{EBO}=\frac{1}{2} \sum_{p}\left(\eta_{b_{p}}-\eta_{a b_{p}}\right)
$$

based on the occupation numbers $\eta$ of bonding (b) and anti-bonding (ab) natural orbitals (NOs). Equation (1) allows for fractional bond orders as the orbital contributions per bond in general will be non-integer. Hence, the multiplicity of a bond is best determined by the lowest integer value larger than the EBO. ${ }^{43}$ In the case of $\mathrm{U}_{2}$, an EBO of 4.2 was obtained and interpreted as a quintuple bond. ${ }^{33}$ Such a translation of an EBO into integer bond multiplicity remains subject to debate as a recent discussion of the bond order in $\mathrm{C}_{2}$ clearly illustrates, see for example Refs. 44 and 45 and references therein.

It is known that not only scalar-relativistic effects, but also SO interactions are expected to strongly influence the chemical bonding and reactivity in heavy-element containing molecular systems. ${ }^{46}$ Recent examples include mercury- ${ }^{47}$ and gold-catalyzed ${ }^{48}$ 
reactions where significant SO coupling effects make accessible catalytic pathways which would otherwise be closed for energetic and/or symmetry reasons. Given the complex nature of the chemical bond in $\mathrm{U}_{2}$, obvious questions that remain are: (i) Whether a variational inclusion of $\mathrm{SO}$ interaction in the wave function optimization could lead to a change of the bonding picture, i.e., is the U-U bond a quintuple bond or not? What is the impact of the SO interaction on the nature of the electronic ground state? A detailed understanding of the electronic structure and the chemical bond in $\mathrm{U}_{2}$ will be helpful to gain further insight into the chemical bonding properties of the $5 f$-elements, and in particular the role of SO interaction. To address these questions, we carried out multiconfigurational CASSCF with the same active space as in Refs. 32 and 33 as well as extended restricted active space (RASSCF) calculations with a variational inclusion of scalar-relativistic effects and SO interaction, using the exact 2-component Hamiltionian (X2C) framework in the DIRAC program package ${ }^{49}$ (see Supplementary Information for computational details). It is worthwhile to note that our RAS model comprises the full set of valence molecular orbitals arising from the $5 f, 6 d$ and $7 s$ manifold of each uranium atom. In addition to the CAS model, it includes all excitations from the strongly bonding $\sigma_{\mathrm{g}}$ and $\pi_{\mathrm{u}}$ orbitals into the anti-bonding $\sigma_{\mathrm{u}}^{*}$ and $\pi_{\mathrm{g}}^{*}$ orbitals (scalar-relativistic notation) plus singles-doubles to the CAS model space for a partial description of dynamic correlation. The RAS model can correctly describe the atomic states at dissociation. Supplementary Figure 1 summarizes both active space models in terms of the actual, considered valence orbitals. By comparison with the corresponding scalar-relativistic CASSCF data reported previously ${ }^{32,33}$ differences in the bonding picture of $\mathrm{U}_{2}$ can unambiguously be ascribed to a variational account of SO interaction.

\section{RESULTS AND DISCUSSIONS}

\section{Effective bond order: generalization}

The calculation of the EBO (cf. equation (1)) hinges on being able to distinguish bonding, anti-bonding and non-bonding orbitals. Without variational account of SO interaction bonding and anti-bonding MOs are easily identified from symmetry in a homonuclear dimer such as $\mathrm{U}_{2}$, as illustrated on the left-hand side in Supplementary Fig. 3. Upon in- 
clusion of SO interaction bonding and anti-bonding orbitals will mix ${ }^{50}$, as shown on the right-hand side in Supplementary Fig. 3. For instance, the bonding spin orbital $\sigma_{g} \alpha$ and anti-bonding $\pi_{g} \beta$ may mix, since they have the same value $m_{j}$ of the projection of total angular momentum on the internuclear axis. This makes a definite characterization of a given MO as bonding or anti-bonding impossible. Obviously the same considerations hold for NOs. Maurice et al. noted that an alternative to equation (1) is to multiply the bond order of individual spin-free configuration state functions (or determinants), obtained by simple counting of bonding and anti-bonding orbitals, by their weight in the total wave function $^{51}$. This allowed them to extend the definition of EBO to multireference calculations starting from a set of molecular orbitals optimized at the scalar relativistic level and with SO interaction only added at the correlated level. Since SO interaction in our case is also present in the orbital optimization step a further generalization of the EBO is required. Hence, we propose a generalized EBO $(g \mathrm{EBO})$,

$$
g \mathrm{EBO}=\frac{1}{2} \sum_{p} \eta_{p}\left(w_{b}-w_{a b}\right),
$$

where we introduced the difference of the weights $w_{b}$ and $w_{a b}$ of the bonding and antibonding contribution to the $p$-th NO, respectively, to be multiplied with the occupation number $\eta_{p}$. Without SO coupling the weights are either zero or one and equation (2) reduces to the EBO definition given in equation (1).

\section{Nature of low-lying electronic states}

In Fig. 2, we present our calculated CASSCF potential energy curves for the lowest electronic states of $\mathrm{U}_{2}$ around the equilibrium structure. The inset in Fig. 2 focuses on internuclear $\mathrm{U}-\mathrm{U}$ distances near the equilibrium of the lowest three electronic states obtained with a larger basis set (dyall.cv3z). These electronic states have term symbols $\Omega=9_{\mathrm{g}}, 8_{\mathrm{g}}$, and $7_{\mathrm{g}}$ (Hund's case (c)) with $\Omega=9_{\mathrm{g}}$ being the lowest state, separated by approximately $2200 \mathrm{~cm}^{-1}$ from the first two excited $\Omega=8_{\mathrm{g}}$ and $\Omega=7_{\mathrm{g}}$ states. Its calculated equilibrium distance and harmonic vibrational frequency are $\mathrm{R}_{e}=2.57 \AA$ and $\omega_{e}=190 \mathrm{~cm}^{-1}$, respectively. Inclusion of more dynamic electron correlation through RASSCF calculations (see Supplementary Fig. 2) confirmed the $\Omega=9_{\mathrm{g}}$ state as electronic ground state of $\mathrm{U}_{2}$. Compared to the CASSCF data, the spectroscopic constants change 
very little $\left(\Delta \mathrm{R}_{e}=-0.01 \AA\right.$ and $\left.\Delta \omega_{e}=-4 \mathrm{~cm}^{-1}\right)$. In contrast, the $\Omega=8_{\mathrm{g}}$ state, which was reported as electronic ground state in previous works ${ }^{32,33}$, comes out as a low-lying excited state about $3500 \mathrm{~cm}^{-1}\left(2200 \mathrm{~cm}^{-1}\right)$ vertically above the $\Omega=9_{\mathrm{g}}$ state, according to our RASSCF (CASSCF) calculations. Moreover, from the RASSCF data we calculated a dissociation energy of $21.0 \mathrm{kcal} / \mathrm{mol}$ from the $\Omega=9_{\mathrm{g}}$ state to two separated $\mathrm{U}$ atoms. Although being approximately $10 \mathrm{kcal} / \mathrm{mol}$ lower than the corresponding ground-state bond energy obtained from SO CASPT2 calculations $^{32}$, this result still supports a bound $\mathrm{U}_{2}$ molecule with a sizable bond energy. It should be noted that, in contrast to the SO CASPT2 approach, our RASSCF model only partially takes into account dynamical electron correlation effects, which could to a large extent explain the observed discrepancy in the dissociation energy.

\section{Bonding in $\mathrm{U}_{2}$}

An analysis of the electronic configuration of the excited $\Omega=8_{\mathrm{g}}$ state (see Table 1) shows excellent agreement with the corresponding data for the spin-free ${ }^{7} \mathrm{O}_{\mathrm{g}}$ ground state reported in Refs. 32 and 33. As discussed by the latter authors, the ${ }^{7} \mathrm{O}_{g}$ spin-free state is the main component of their SO coupled $\Omega=8_{\mathrm{g}}$ state. Our analysis in Table 1 confirms that the chemical bond in the $\Omega=8_{\mathrm{g}}$ state can be regarded as consisting of four (weak) one-electron two-center bonds with contributions from $6 d$ and $5 f$ AOs as well as two non-bonding $5 f \mathrm{MOs}^{32}$. The reorganization of the electronic valence configuration of each $\mathrm{U}$ atom in the molecule is shown by the Mulliken atomic orbital populations given in Supplementary Table 2. Each $\mathrm{U}$ atom has a $7 s$ population around 1.0 and a $6 d$ population somewhat above 2.5, suggesting the $7 s \rightarrow 6 d$ promotion discussed above. By contrast, each $U$ atom retains to a large extent its atomic $5 f^{3}$ ground state occupation such that any $5 f \rightarrow 7 s$ promotion is modest, as would be expected from a consideration of promotion energies (cf. Ref. 33).

The Mulliken atomic orbital populations per $\mathrm{U}$ atom for the $\Omega=9_{g}$ ground state are qualitatively similar to those of the $\Omega=8_{\mathrm{g}}$ excited state. In contrast, the NO occupation numbers listed in Table 1 reveal a notable difference between the electronic configurations of these states. Irrespective of the active space models, distinctive features of the $\Omega=9_{\mathrm{g}}$ state in comparison to the $\Omega=8_{\mathrm{g}}$ state are its singly occupied orbitals of predominantly 
$5 f_{\delta}$-type and empty orbitals with mainly $5 f_{\pi}$-character, originating from distinctively different leading determinants at the spin-free level. Table 2 summarizes the decomposition of the exact total angular momentum $\Omega$ on the internuclear axis into its averaged angular momentum $\Lambda$ and spin momentum $\Sigma$ contributions (see Supplementary Information for computational definitions). Considering first the $\Omega=8_{\mathrm{g}}$ state, we note that the spin projection expectation value $\langle\Sigma\rangle$ for either active space model $--2.51(-2.60)$ for CASSCF (RASSCF) - is non-integer and close to halfway between the possible non- or scalarrelativistic integer values (in absolute terms) of two and three. For the $\Omega=9_{\mathrm{g}}$ state, we observe a decrease of the total spin projection $\Sigma$ to $-2.67(-2.79)$ for the CASSCF (RASSCF) active space model. These non-integer values are consistent with a mixing of spin-free states with $\mathrm{M}_{S}=-2$ and $\mathrm{M}_{S}=-3$ both originating from septet $(S=3)$ states, or, alternatively, with a mixing of quintet and septet spin-free states. The latter case would imply that SO coupling reduces the ferromagnetic character of the coupling of the six unpaired electrons in both states ${ }^{33}$. We can, however, not provide a sound confirmation of this since our calculations do not contain intermediate spin-free states. Interestingly, a full antiferromagnetic coupling of the unpaired electrons was reported for the electronic ground state configuration of metastable $\mathrm{U}_{2}^{2+}{ }^{52}$ We have confirmed this picture in $\mathrm{U}_{2}^{2+}$ for our $\Omega=1_{\mathrm{g}}$ ground $(\Sigma=-0.08)$ and $\Omega=0_{\mathrm{g}}^{+}(\Sigma=0.00)$ excited states whereas other electronically excited states shown in Supplementary Fig. 5 with $\Omega>1$ exhibit larger departures from integer $\Sigma$ values.

In Table 2 we also report $g$ EBOs for the $\Omega=9_{\mathrm{g}}$ and $\Omega=8_{\mathrm{g}}$ states obtained according to equation (2). They are lower than four for either electronic state (and active orbital space model employed) at their respective equilibrium structures with a common lowering of the $g$ EBO by approximately 0.1 in going from our CAS- to RASSCF active orbital space model. These findings strongly suggest the presence of a quadruple rather than the quintuple bond proposed in previous works ${ }^{32,33}$. As shown in Supplementary Fig. 4, these conclusions even hold for a larger range of U-U distances, thus a change in bond lengths by full account of dynamic correlations will not change the conclusions. 


\section{CONCLUSIONS}

We have calculated and characterized the low-lying electronic spectrum of $\mathrm{U}_{2}$ by means of a genuine relativistic multiconfigurational quantum chemical approach with full variational inclusion of spin-orbit effects. In summary, our best calculations predict an $\Omega=9_{\mathrm{g}}$ ground state separated by $3500 \mathrm{~cm}^{-1}$ from the first excited state, which we identified as an $\Omega=8$ g state, which is the lowest in a manifold of many low-lying excited states, as illustrated in Fig. 2. Furthermore, the ground state is predicted to have an equilibrium distance of $R_{e}=2.56 \AA$, a harmonic vibrational frequency of $\omega_{e}=186 \mathrm{~cm}^{-1}$, and a dissociation energy of $D_{e}>21 \mathrm{kcal} / \mathrm{mol}$.

Our calculated data reveal essential differences compared to previous calculations in the description of the complex electronic structure of $\mathrm{U}_{2}$, differences that ultimately call for a revision of the proposed chemical bond picture for the $\mathrm{U}_{2}$ ground state established in previous works ${ }^{32,33}$ : (i) the electronic ground state has term symbol $\Omega=9_{\mathrm{g}}$ and is characterized by a generalized effective bond order of 3.8 which translates into a quadruple bond. (ii) The $\Omega=8_{\mathrm{g}}$ state which was proposed to be the electronic ground state of $\mathrm{U}_{2}$ with an effective bond order of 4.2 (corresponding to a quintuple bond) ${ }^{32,33}$ is in fact a low-lying electronically excited state with a quadruple bond (generalized effective bond order of 3.9) at its equilibrium structure. Our analysis shows that the chemical bond in the $\Omega=9_{\mathrm{g}}$ electronic ground state consists of three electron-pair bonds, one $\sigma$ and two $\pi$-bonds, two one-electron bonds of approximate $\sigma$-and $\delta$-type as well as four predominantly ferromagnetically coupled $5 f$ electrons localized on one $\mathrm{U}$ atom each. This distinctly simplifies not only the originally proposed bonding picture ${ }^{32}$ but also emphasizes the value of a genuine relativistic multiconfigurational electron correlation approach that facilitates an unambiguous prediction of the electronic structure and molecular properties of molecules containing heavy elements such as uranium.

\section{Data availability}

Supplementary information is available as a separate file. All relevant data that are not included in this Article and its Supplementary Information are available from the corresponding author upon reasonable request. 


\section{Acknowledgements}

We thank the Danish Center for Scientific Computing for ample computational resources. We thank Trygve Helgaker and Einar Uggerud (U Oslo) for help with Ref. 34. We thank Camilla Kottum Elmar for preparing the graphical abstract. SK acknowledges fruitful discussions with Pekka Pyykkö (U Helsinki) on numerous occasions. SK thanks Markus Reiher (ETH Zürich) for his continuous support. We dedicate this paper to the memory of Björn Roos, an outstanding quantum chemist.

\section{Author contributions}

SK ran all calculations reported in this paper, whereas the interpretation of results as well as preparation of the manuscript was a joint effort by all three authors. The generalized effective bond order (equation (2)) was developed by SK and TS.

\section{Competing interests}

The authors declare no competing financial interests.

\section{Corresponding authors}

Correspondence to Stefan Knecht.

\section{REFERENCES}

${ }^{1}$ Lewis, G. N. The atom and the molecule. J. Am. Chem. Soc. 38, 762-785 (1916).

${ }^{2}$ Neidig, M. L., Clark, D. L. \& Martin, R. L. Covalency in f-element complexes. Coord. Chem. Rev. 257, 394-406 (2013).

${ }^{3}$ Kaltsoyannis, N. \& Kerridge, A. Chemical bonding of lanthanides and actinides. In Frenking, G. \& Shaik, S. (eds.) The chemical bond: chemical bonding across the periodic table, 337-355 (Wiley, Weinheim, 2014).

${ }^{4}$ Cary, S. K. et al. Emergence of californium as the second transitional element in the actinide series. Nat. Commun. 6, 6827 (2015). 
${ }^{5}$ Ferrier, M. G. et al. Spectroscopic and computational investigation of actinium coordination chemistry. Nat. Commun. 7, 12312 (2016).

${ }^{6}$ Dutkiewicz, M. S., Apostolidis, C., Walter, O. \& Arnold, P. L. Reduction chemistry of neptunium cyclopentadienide complexes: from structure to understanding. Chem. Sci. 8, 2553-2561 (2017).

${ }^{7}$ Windorff, C. J. et al. Identification of the formal +2 oxidation state of plutonium: synthesis and characterization of $\left\{\mathrm{Pu}^{\mathrm{II}}\left[\mathrm{C}_{5} \mathrm{H}_{3}\left(\mathrm{SiMe}_{3}\right)_{2}\right]_{3}\right\}^{-}$. J. Am. Chem. Soc. 139, 3970-3973 (2017).

${ }^{8}$ Jung, J., Atanasov, M. \& Neese, F. Ab initio ligand-field theory analysis and covalency trends in actinide and lanthanide free ions and octahedral complexes. Inorg. Chem. 56, $8802-8816$ (2017).

${ }^{9}$ Cross, J. N. et al. Covalency in americium(III) hexachloride. J. Am. Chem. Soc. 139, 8667-8677 (2017).

${ }^{10}$ Formanuik, A. et al. Actinide covalency measured by pulsed electron paramagnetic resonance spectroscopy. Nat. Chem. 9, 578-583 (2017).

${ }^{11}$ Vitova, T. et al. The role of the $5 \mathrm{f}$ valence orbitals of early actinides in chemical bonding. Nat. Commun. 8, 16053 (2017).

${ }^{12}$ Wilson, R. E., De Sio, S. \& Vallet, V. Protactinium and the intersection of actinide and transition metal chemistry. Nat. Commun. 9, 622 (2018).

${ }^{13}$ Editorial. The bottom line. Nat. Chem. 9, 831 (2017).

${ }^{14}$ Monreal, M. J. \& Diaconescu, P. L. The riches of uranium. Nat. Chem. 2, 424 (2010).

${ }^{15} \mathrm{Li}$ Manni, G. et al. Assessing metal-metal multiple bonds in Cr-Cr, Mo-Mo, and W-W compounds and a hypothetical U-U compound: A quantum chemical study comparing DFT and multireference methods. Chem. Eur. J. 18, 1737-1749 (2012).

${ }^{16}$ Penchoff, D. A. \& Bursten, B. E. Metal-metal bonding in the actinide elements: Conceptual synthesis of a pure two-electron $\mathrm{U}-\mathrm{U}_{\delta}$ single bond in a constrained geometry of $\mathrm{U}_{2}(\mathrm{OH})_{10}$. Inorg. Chim. Acta 424, 267-273 (2015).

${ }^{17}$ Foroutan-Nejad, C., Vicha, J., Marek, R., Patzschke, M. \& Straka, M. Unwilling U$\mathrm{U}$ bonding in $\mathrm{U}_{2} @ \mathrm{C}_{80}$ : cage-driven metal-metal bonds in di-uranium fullerenes. Phys. Chem. Chem. Phys. 17, 24182-24192 (2015). 
${ }^{18}$ Qu, N., Su, D.-M., Wu, Q.-Y., Shi, W.-Q. \& Pan, Q.-J. Metal-metal multiple bond in low-valent diuranium porphyrazines and its correlation with metal oxidation state: A relativistic DFT study. Comput. Theor. Chem. 1108, 29-39 (2017).

${ }^{19}$ Zhang, X. et al. $\mathrm{U}_{2} @ \mathrm{I}_{h}(7)-\mathrm{C}_{80}$ : Crystallographic characterization of a long-sought dimetallic actinide endohedral fullerene. J. Am. Chem. Soc. 140, 3907-3915 (2018).

${ }^{20}$ Scheibe, B. et al. The $\left[\mathrm{U}_{2} \mathrm{~F}_{12}\right]^{2-}$ anion of $\mathrm{Sr}\left[\mathrm{U}_{2} \mathrm{~F}_{12}\right]$. Angew. Chem. Int. Ed. 57, 29142918 (2018).

${ }^{21}$ Lyon, J. T., Hu, H.-S., Andrews, L. \& Li, J. Formation of unprecedented actinide $\equiv$ carbon triple bonds in uranium methylidyne molecules. Proc. Nat. Acad. Sci. 104, 18919-18924 (2007).

${ }^{22}$ Hu, H.-S., Qiu, Y.-H., Xiong, X.-G., Schwarz, W. H. E. \& Li, J. On the maximum bond multiplicity of carbon: unusual C-U quadruple bonding in molecular CUO. Chem. Sci. 3, 2786-2796 (2012).

${ }^{23}$ Hayton, T. W. Recent developments in actinide-ligand multiple bonding. Chem. Comm. 49, 2956 (2013).

${ }^{24}$ Hlina, J. A., Pankhurst, J. R., Kaltsoyannis, N. \& Arnold, P. L. Metal-metal bonding in uranium-group 10 complexes. J. Am. Chem. Soc. 138, 3333-3345 (2016).

${ }^{25}$ Ephritikhine, M. Molecular actinide compounds with soft chalcogen ligands. Coord. Chem. Rev. 319, 35-62 (2016).

${ }^{26}$ Fox, A. R., Bart, S. C., Meyer, K. \& Cummins, C. C. Towards uranium catalysts. Nature 455, 341-349 (2008).

${ }^{27}$ Liddle, S. T. The renaissance of non-aqueous uranium chemistry. Angew. Chem. Int. Ed. 54, 8604-8641 (2015).

${ }^{28}$ Halter, D. P., Heinemann, F. W., Bachmann, J. \& Meyer, K. Uranium-mediated electrocatalytic dihydrogen production from water. Nature 530, 317-321 (2016).

${ }^{29}$ Arnold, P. L. \& Turner, Z. R. Carbon oxygenate transformations by actinide compounds and catalysts. Nat. Rev. Chem. 1, 0002 (2017).

${ }^{30}$ Bursten, B. E. \& Ozid, G. A. X $\alpha$-SW calculations for naked actinide dimers: on the existence of $\phi$ bonds between metal atoms. Inorg. Chem. 23, 2910-2911 (1984).

${ }^{31}$ Pepper, M. \& Bursten, B. E. Ab initio studies of the electronic structure of the diuranium molecule. J. Am. Chem. Soc. 112, 7803-7804 (1990). 
${ }^{32}$ Gagliardi, L. \& Roos, B. Quantum chemical calculations show that the uranium molecule $\mathrm{U}_{2}$ has a quintuple bond. Nature 433, 848-851 (2005).

${ }^{33}$ Roos, B. O., Malmqvist, P.-A. \& Gagliardi, L. Exploring the actinide-actinide bond: theoretical studies of the chemical bond in $\mathrm{Ac}_{2}, \mathrm{Th}_{2}, \mathrm{~Pa}_{2}$, and $\mathrm{U}_{2}$. J. Am. Chem. Soc. 128, 17000-17006 (2006).

${ }^{34}$ Gorokhov, L. N., Emelyanov, A. M. \& Khodeev, Y. S. Mass-spectroscopic investigation of stability of gaseous $\mathrm{U}_{2} \mathrm{O}_{2}$ and $\mathrm{U}_{2}$. High. Temp. 12, 1307-1309 (1974).

${ }^{35}$ Pyykkö, P. \& Desclaux, J.-P. Relativity and the periodic system of elements. Acc. Chem. Res. 12, 276-281 (1979).

${ }^{36}$ Reiher, M. \& Wolf, A. Relativistic quantum chemistry: the fundamental theory of molecular science (Wiley, Weinheim, Weinheim, 2009).

${ }^{37}$ Dyall, K. G. \& Fægri, K. Introduction to Relativistic Quantum Chemistry (Oxford University Press, Oxford, 2007).

${ }^{38}$ Saue, T. Relativistic Hamiltonians for chemistry: a primer. ChemPhysChem 12, 30773094 (2011).

${ }^{39}$ Christiansen, P. A. \& Pitzer, K. S. Electronic structure and dissociation curves for the ground states of $\mathrm{Tl}_{2}$ and $\mathrm{Tl}_{2}^{+}$from relativistic effective potential calculations. J. Chem. Phys. 74, 1162-1165 (1981).

${ }^{40}$ Saue, T., Fægri, K. \& Gropen, O. Relativistic effects on the bonding of heavy and superheavy hydrogen halides. Chem. Phys. Lett. 263, 360-366 (1996).

${ }^{41}$ Blaise, J. \& Wyart, J. F. Selected constants energy levels and atomic spectra of actinides (Centre National de la Recherche Scientifique, France, 1992). See also http://web2. lac.u-psud.fr/lac/Database/Contents.html.

${ }^{42}$ Herzberg, G. Zum Aufbau der zweiatomigen Moleküle. Z. Phys. 57, 601-630 (1929).

${ }^{43}$ Roos, B. O., Borin, A. C. \& Gagliardi, L. Reaching the maximum multiplicity of the covalent chemical bond. Angew. Chem. Int. Ed. 46, 1469-1472 (2007).

${ }^{44}$ Shaik, S. et al. Quadruple bonding in $\mathrm{C}_{2}$ and analogous eight-valence electron species. Nat. Chem. 4, 195-200 (2012).

${ }^{45}$ Shaik, S., Rzepa, H. S. \& Hoffmann, R. One molecule, two atoms, three views, four bonds? Angew. Chem. Int. Ed. 52, 3020-3033 (2013). 
${ }^{46}$ Schwerdtfeger, P. Relativity and chemical bonding. In Frenking, G. \& Shaik, S. (eds.) The chemical bond: fundamental aspects of chemical bonding, 383-404 (Wiley, Weinheim, 2014).

${ }^{47}$ Demissie, T. B., Garabato, B. D., Ruud, K. \& Kozlowski, P. M. Mercury methylation by cobalt corrinoids: relativistic effects dictate the reaction mechanism. Angew. Chem. Int. Ed. 55, 11503-11506 (2016).

${ }^{48}$ Gaggioli, C. A. et al. Dioxygen insertion into the gold(I)-hydride bond: spin orbit coupling effects in the spotlight for oxidative addition. Chem. Sci. 7, 7034-7039 (2016). ${ }^{49}$ DIRAC, a relativistic ab initio electronic structure program, Release DIRAC17 (2017), written by L. Visscher, H. J. Aa. Jensen, R. Bast, and T. Saue, with contributions from V. Bakken, K. G. Dyall, S. Dubillard, U. Ekström, E. Eliav, T. Enevoldsen, E. Faßhauer, T. Fleig, O. Fossgaard, A. S. P. Gomes, E. D. Hedegård, T. Helgaker, J. Henriksson, M. Iliaš, Ch. R. Jacob, S. Knecht, S. Komorovský, O. Kullie, J. K. Lærdahl, C. V. Larsen, Y. S. Lee, H. S. Nataraj, M. K. Nayak, P. Norman, G. Olejniczak, J. Olsen, J. M. H. Olsen, Y. C. Park, J. K. Pedersen, M. Pernpointner, R. di Remigio, K. Ruud, P. Sałek, B. Schimmelpfennig, A. Shee, J. Sikkema, A. J. Thorvaldsen, J. Thyssen, J. van Stralen, S. Villaume, O. Visser, T. Winther, and S. Yamamoto (see http://www.diracprogram.org).

${ }^{50}$ Balasubramanian, K. Relativity and chemical bonding. J. Phys. Chem. 93, 6585-6596 (1989).

${ }^{51}$ Maurice, R. et al. Effective bond orders from two-step spin-orbit coupling approaches: the $\mathrm{I}_{2}, \mathrm{At}_{2}, \mathrm{IO}^{+}$, and $\mathrm{AtO}^{+}$case studies. J. Chem. Phys. 142, 094305 (2015).

${ }^{52}$ Gagliardi, L., Pyykkö, P. \& Roos, B. O. A very short uranium-uranium bond: the predicted metastable $\mathrm{U}_{2}^{2+}$. Phys. Chem. Chem. Phys. 7, 2415-2417 (2005).

${ }^{53}$ Jong, W. A. D., Visscher, L. \& Nieuwpoort, W. C. On the bonding and the electric field gradient of the uranyl ion. J. Mol. Struct. THEOCHEM 458, 41-52 (1999). Corrigendum 581:259, 2002.

${ }^{54}$ Dyall, K. G. Relativistic double-zeta, triple-zeta, and quadruple-zeta basis sets for the actinides Ac-Lr. Theor. Chem. Acc. 117, 491-500 (2007). 


\section{Figure captions}

Figure 1. Frontier atomic valence orbital energies of uranium in nonrelativistic (left), scalarrelativistic (middle), and fully relativistic (right) molecular orbital theories. All valence orbitals of uranium, $5 f, 6 d$ and $7 s$ orbitals are (partially) occupied in the electronic ground-state configuration. Relativistic effects are substantial for the heavy element $\mathrm{U}$, leading to an energetic reordering of the $7 s$ (red lines) and $6 d$ shells (blue lines) while bringing the $5 f$ shell (green lines) energetically closer to the remaining valence shells. The importance of spin-orbit coupling is readily seen by the notable energetic splitting of the valence orbitals with angular momentum number $l>0$, i.e., the $5 f$ and $6 d$ orbitals, leading to their increased availability for bonding. Orbital energies were obtained from atomic average-of-configuration Hartree-Fock calculations (see Supplementary Information for further computational details).

Figure 2. CASSCF potential energy curves for the lowest electronic states of $\mathrm{U}_{2}$ around the equilibrium structure calculated with the uncontracted $[26 s 21 p 17 d 12 f 3 g]$ deJong $+3 g$ basis set ${ }^{53}$. The inset shows the CASSCF potential energy curves for the first three electronic states of $\mathrm{U}_{2}$ around the equilibrium structure calculated with the uncontracted [33s29p20d15f6g2h] dyall.cv3z basis set ${ }^{54}$ with identical color and symbol codes for the electronic states as in the main figure. The lowest-lying electronic states have term symbols $\Omega=9_{\mathrm{g}}$ (red circles), $8_{\mathrm{g}}$ (green circles), and $7_{\mathrm{g}}$ (purple circles) (term designation in accordance with Hund's case (c)). In both cases, $\Omega=9_{\mathrm{g}}$ is the lowest state with a notable vertical separation of more than $2000 \mathrm{~cm}^{-1}$ from the remaining states. The $\Omega=8$ g state, reported as electronic ground state in previous works ${ }^{32,33}$, is found to be among the first excited states. Electronic states with an $\Omega$ quantum number lower than 7 and those with ungerade inversion symmetry are even higher-lying in energy in comparison to the $\Omega=9_{\mathrm{g}}$ state. An exception is the $\Omega=7_{\mathrm{u}}$ state which becomes the lowest-lying excited state at larger U-U internuclear distances. Absolute energies are reported with an offset of -56075 Hartree. 


\section{Tables}

Table 1. Electronic configurations for $\mathrm{U}_{2}$ in different electronic states at their respective equilibrium structure. For the relativistic results including spin-orbit coupling, the natural orbitals are labeled according to their main scalar-relativistic character which eases comparison with the reference data of Ref. 32. Natural orbitals with occupancies higher than 0.1 in all configurations are listed.

\begin{tabular}{lc}
\hline \hline & $\Omega=8_{\mathrm{g}}$ \\
$\mathrm{CASSCF}$ & $7 s \sigma_{g}^{2.00} 6 d \pi_{u}^{4.00} 6 d \sigma_{g}^{1.03} 6 d \delta_{g}^{0.97} 5 f \pi_{g}^{0.38} 5 f \pi_{u}^{0.58} 5 f \delta_{g}^{0.57} 5 f \delta_{u}^{0.42} 5 f \phi_{g}^{1.00} 5 f \phi_{u}^{1.00}$ \\
$\mathrm{RASSCF}$ & $7 s \sigma_{g}^{1.91} 6 d \pi_{u}^{3.80} 6 d \sigma_{g}^{0.97} 6 d \delta_{g}^{0.97} 5 f \pi_{g}^{0.41} 5 f \pi_{u}^{0.59} 5 f \delta_{g}^{0.59} 5 f \delta_{u}^{0.41} 5 f \phi_{g}^{0.99} 5 f \phi_{u}^{1.00}$ \\
$\mathrm{CASSCF}^{a 32,33}$ & $7 s \sigma_{g}^{2.00} 6 d \pi_{u}^{4.00} 6 d \sigma_{g}^{0.97} 6 d \delta_{g}^{0.98} 5 f \pi_{g}^{0.37} 5 f \pi_{u}^{0.63} 5 f \delta_{g}^{0.63} 5 f \delta_{u}^{0.37} 5 f \phi_{g}^{1.00} 5 f \phi_{u}^{1.00}$ \\
\multicolumn{1}{c}{$\Omega=9_{\mathrm{g}}$} \\
$\mathrm{CASSCF}$ \\
$\mathrm{RASSCF}$ & $7 s \sigma_{g}^{2.00} 6 d \pi_{u}^{4.00} 6 d \sigma_{g}^{0.96} 6 d \delta_{g}^{0.97} 5 f \pi_{g}^{0.00} 5 f \pi_{u}^{0.00} 5 f \delta_{g}^{1.00} 5 f \delta_{u}^{0.99} 5 f \phi_{g}^{1.00} 5 f \phi_{u}^{1.00}$ \\
\hline
\end{tabular}

${ }^{a}$ Obtained from state-interaction calculations of the ${ }^{7} \mathrm{O}_{\mathrm{g}}$ spin-free state with several electronic states of different spin multiplicities via the spin-orbit coupling Hamiltonian.

Table 2. Decomposition of the total angular momentum $\Omega$ of the $\Omega=8_{\mathrm{g}}$ and $9_{\mathrm{g}}$ electronic states of $\mathrm{U}_{2}$. In addition, we report $g$ EBOs calculated according to equation (2).

\begin{tabular}{lccccccc}
\hline \hline Method & $\langle\Lambda\rangle$ & $\langle\Sigma\rangle$ & $g \mathrm{EBO}$ & & $\langle\Lambda\rangle$ & $\langle\Sigma\rangle$ & $g \mathrm{EBO}$ \\
\hline & \multicolumn{3}{c}{$\Omega=8_{\mathrm{g}}$} & & \multicolumn{3}{c}{$\Omega=9_{\mathrm{g}}$} \\
\cline { 2 - 5 } \cline { 5 - 8 } CASSCF & 10.51 & -2.51 & 3.9 & & 11.67 & -2.67 & 3.8 \\
RASSCF & 10.60 & -2.60 & 3.8 & & 11.79 & -2.79 & 3.7 \\
CASSCF [Ref. 33] & 11 & -3 & $4.2^{a}$ & & 12 & -3 & - \\
\hline
\end{tabular}

${ }^{a}$ EBO data. 